\title{
Effect of Concussion on Clinically Measured Reaction Time in 9 NCAA Division I Collegiate Athletes: A Preliminary Study
}

\author{
James T. Eckner, MD, MS, Jeffrey S. Kutcher, MD, James K. Richardson, MD
}

Objectives: To evaluate the effect of concussion on clinically measured reaction time $\left(\mathrm{RT}_{\text {clin }}\right)$ and in comparison to a computerized reaction time measure $\left(\mathrm{RT}_{\text {comp }}\right)$.

Design: Prospective, repeated measures observational study.

Setting: Athletic training clinic at a National Collegiate Athletic Association (NCAA) Division I university.

Participants: Data are reported for 9 collegiate athletes with acute concussion who were part of a larger cohort of 209 athletes recruited from the university's football, women's soccer, and wrestling teams before the start of their respective athletic seasons.

Methods: Baseline $\mathrm{RT}_{\text {clin }}$ and $\mathrm{RT}_{\text {comp }}$ were measured during preparticipation physical examinations. $\mathrm{RT}_{\text {clin }}$ measured the time required to catch a suspended vertical shaft by hand closure after its release by the examiner. $\mathrm{RT}_{\text {comp }}$ was derived from the simple RT component of the CogState-Sport computerized neurocognitive test battery. Athletes who subsequently sustained a physician-diagnosed concussion underwent repeated $\mathrm{RT}_{\text {clin }}$ and $\mathrm{RT}_{\text {comp }}$ testing within 72 hours of injury. A Wilcoxon signed rank test was used to compare baseline and after-injury RTs.

Main Outcome Measurements: After-injury changes in $\mathrm{RT}_{\text {clin }}$ and $\mathrm{RT}_{\text {comp }}$ were calculated with respect to each athlete's own preseason baseline value.

Results: After-injury $\mathrm{RT}_{\text {clin }}$ was prolonged in 8 of the 9 athletes with concussions, whereas $\mathrm{RT}_{\text {comp }}$ was prolonged in 5 of the 9 athletes with concussions. The mean (standard deviation) $\mathrm{RT}_{\text {clin }}$ increased from $193 \pm 21 \mathrm{~ms}$ to $219 \pm 31 \mathrm{~ms}(P=.050)$, and mean $\mathrm{RT}_{\text {comp }}$ increased from $247 \pm 75$ to $462 \pm 120 \mathrm{~ms}(P=.214)$.

Conclusions: We concluded that $\mathrm{RT}_{\text {clin }}$ appears to be sensitive to the known prolongation of RT after concussion and compares favorably with an accepted computerized RT measure. This study supports the potential utility of $\mathrm{RT}_{\text {clin }}$ as part of a multifaceted concussion assessment battery.

PM R 2011;3:212-218

\section{INTRODUCTION}

Sports-related concussion is a common and serious injury. Recent estimates from the Centers for Disease Control and Prevention are that approximately 1.6-3.8 million sportsassociated traumatic brain injuries occur annually in the United States, with the vast majority classified as mild traumatic brain injury or concussion [1]. Results of epidemiologic studies have shown that concussions account for 5\%-10\% of all injuries associated with organized sports participation at the high school and collegiate levels [2,3]. Concussions were once considered minor injuries that an athlete could play through without consequence. However, an explosion in the number and quality of concussion research articles published in the scientific literature over the past 20 years coupled with a great deal of recent attention in the popular press have largely changed this attitude. Traumatic brain injury and the issue of determining the readiness of a athlete with a concussion to return to sports participation are now considered major public health issues $[4,5]$. The appropriate diagnosis and management of athletes with concussion remains of primary importance in minimizing the short-term effects of injury on an athlete and preventing poor outcomes that can result from premature return to sports participation. In addition, recently there has been
J.T.E. Department of Physical Medicine and Rehabilitation, University of Michigan, $325 \mathrm{E}$. Eisenhower Pkwy, Ann Arbor, Ml 48108. Address correspondence to J.T.E.; e-mail: jeckner@med.umich.edu

Disclosure: 4A, University of Michigan patent application; 8, Foundation for PM\&R (New Investigator Award); 8B, the University of Michigan Bone and Joint Injury Prevention and Rehabilitation Center (Pilot Grant)

J.S.K. Department of Neurology, University of Michigan, Ann Arbor, Ml Disclosure: nothing to disclose

J.K.R. Department of Physical Medicine and Rehabilitation, University of Michigan, Ann Arbor, MI

Disclosure: 4A, University of Michigan patent application; 8B, University of Michigan Bone and Joint Injury Prevention and Rehabilitation Center (Pilot Grant)

Peer reviewers and all others who control content have no relevant financial relationships to disclose.

Disclosure Key can be found on the Table of Contents and at www.pmrjournal.org

The clinical reaction time device discussed in this article was made by the study authors and is not commercially available. The authors have not applied for medical device status with the U.S. Food and Drug Administration for this device.

Submitted for publication July 16, 2010, accepted December 1, 2010 
increasing attention focused on the potential long-term effects that repeated or mismanaged concussions can have on athletes later in life [6-8].

With these considerations in mind, it is of clear importance that sports medicine providers be able to properly diagnose and manage these injuries. A significant challenge faced by physicians and the other sports medicine providers who evaluate athletes with concussion is that there is no single diagnostic test for concussion. Given the heterogeneous nature of this injury, a multifaceted approach to assessment is essential. All recently published concussion-management guidelines appropriately emphasize the value of such a multifaceted approach [9-11]. The concussion evaluation should assess the presence and severity of typical concussive symptoms and neurologic signs, including balance and cognition. Standardized assessment tools can be helpful because they allow for comparison of after-injury performance of an athlete with concussion to his or her own preseason baseline. One form of standardized assessment tool that has been growing in popularity among the sports medicine community for this purpose is the computerized cognitive assessment battery. Although numerous computerized test programs exist [12-14], all share several features, one of which is the ability to assess reaction time (RT).

Impaired RT is one of the most common cognitive sequelae of concussion. It represents one of the most sensitive indices of cognitive change after concussion $[15,16]$ and has been shown to have prognostic value in predicting time to recovery [17]. RT is typically prolonged immediately after injury, with a gradual return back to baseline during the recovery period [18-24]. Impaired RT generally parallels the presence of other self-reported concussion symptoms $[18,22,25,26]$, but, in some athletes, RT remains impaired even after the athlete has become asymptomatic $[21,23,27]$. Therefore, RT assessment is an important component of the sports medicine provider's concussion assessment battery that can increase the sensitivity of the clinical examination for detecting the effects of sports-related concussion. In addition to its importance as a sign of concussion, impaired RT has functional relevance given that a rapid RT is necessary for injury avoidance. Unfortunately, the dependence of current RT assessment tools on a computer running specialized software limits their utility during the initial sideline concussion assessment and makes them inaccessible to many youth athletes who play in leagues with limited financial resources.

To broaden the availability of RT measurement, we developed a simple visuomotor RT test that can be used on the sideline or in the athletic training clinic. In pilot work, this clinical measure of RT $\left(\mathrm{RT}_{\text {clin }}\right)$ was reliable and valid, with comparable stability from season to season to RT measures currently included in computerized cognitive assessment batteries [28-30]. We also have shown functional relevance of $\mathrm{RT}_{\text {clin }}$, because it is highly correlated with the ability to quickly raise one's hands to protect the head in a simulated sports environment [31]. This study's primary aim was to determine the effect of concussion on $\mathrm{RT}_{\text {clin }}$ by comparing preseason $\mathrm{RT}_{\text {clin }}$ with $\mathrm{RT}_{\text {clin }}$ after a sports-related concussion. A secondary aim was to compare the effect of concussion on $\mathrm{RT}_{\text {clin }}$ with its effect on an accepted and validated computerized measure of RT ( $\left.\mathrm{RT}_{\text {comp }}\right)$.

\section{METHODS}

\section{Subjects}

We recruited, over 2 seasons, 209 student athletes at a single university during preparticipation physical examinations. Members of the football, wrestling, and women's soccer teams who were at least 18 years of age at the time of their preparticipation examination were eligible to participate. Athletes were excluded if they were recovering from an acute concussion or an upper-extremity injury that would prevent them from completing the $\mathrm{RT}_{\text {clin }}$ task. Each athlete provided informed written consent approved by the institutional review board of the University of Michigan before completing baseline $\mathrm{RT}_{\text {clin }}$ and $\mathrm{RT}_{\text {comp }}$ assessments.

\section{Data Collection}

The $\mathrm{RT}_{\text {clin }}$ test protocol used has been described previously $[28,29]$. In brief, the athlete sat with a forearm resting on a desk surface and with the hand at the edge such that it was held sufficiently open to fit around, but not touch, the weighted disk portion of the $\mathrm{RT}_{\text {clin }}$ apparatus (Figure $1 \mathrm{~A}$ ). The apparatus is a rigid $80-\mathrm{cm}$ cylinder coated with highfriction tape, marked in $0.5 \mathrm{~cm}$ increments, and affixed to a weighted disk at one end. The examiner vertically suspended the device with the top of the weighted disk aligned with the superior-most aspect of the athlete's open hand. The examiner released the apparatus after randomly determined delay intervals, between 2 and 5 seconds, to prevent the athlete from anticipating its time of release. Upon release, the athlete caught the device as rapidly as possible by hand closure (Figure 1B). The distance (in $\mathrm{cm}$ ) that the apparatus fell before being caught by the athlete was recorded and used to calculate $\mathrm{RT}_{\text {clin }}$ (in ms) for each trial by using the formula for a body falling under the influence of gravity $\left(\mathrm{d}=0.5 \mathrm{gt}^{2}\right)$. Each athlete performed 2 practice trials, followed by 8 data acquisition trials. Mean (standard deviation [SD]) baseline $\mathrm{RT}_{\text {clin }}$ values were calculated for each athlete.

$\mathrm{RT}_{\text {comp }}$ was derived from each athlete's performance on the simple RT component of CogState-Sport (version 5.6.4; CogState Ltd, Melbourne, Australia) as previously described [28]. CogState-Sport is a computerized neurocognitive assessment battery composed of 4 tasks that assess simple RT, choice RT, learning, and working memory. CogState-Sport also includes a brief self-reported concussion history and concussion symptom assessment by using the Sideline Concussion Assessment Tool (SCAT) [32]. CogState-Sport's re- 

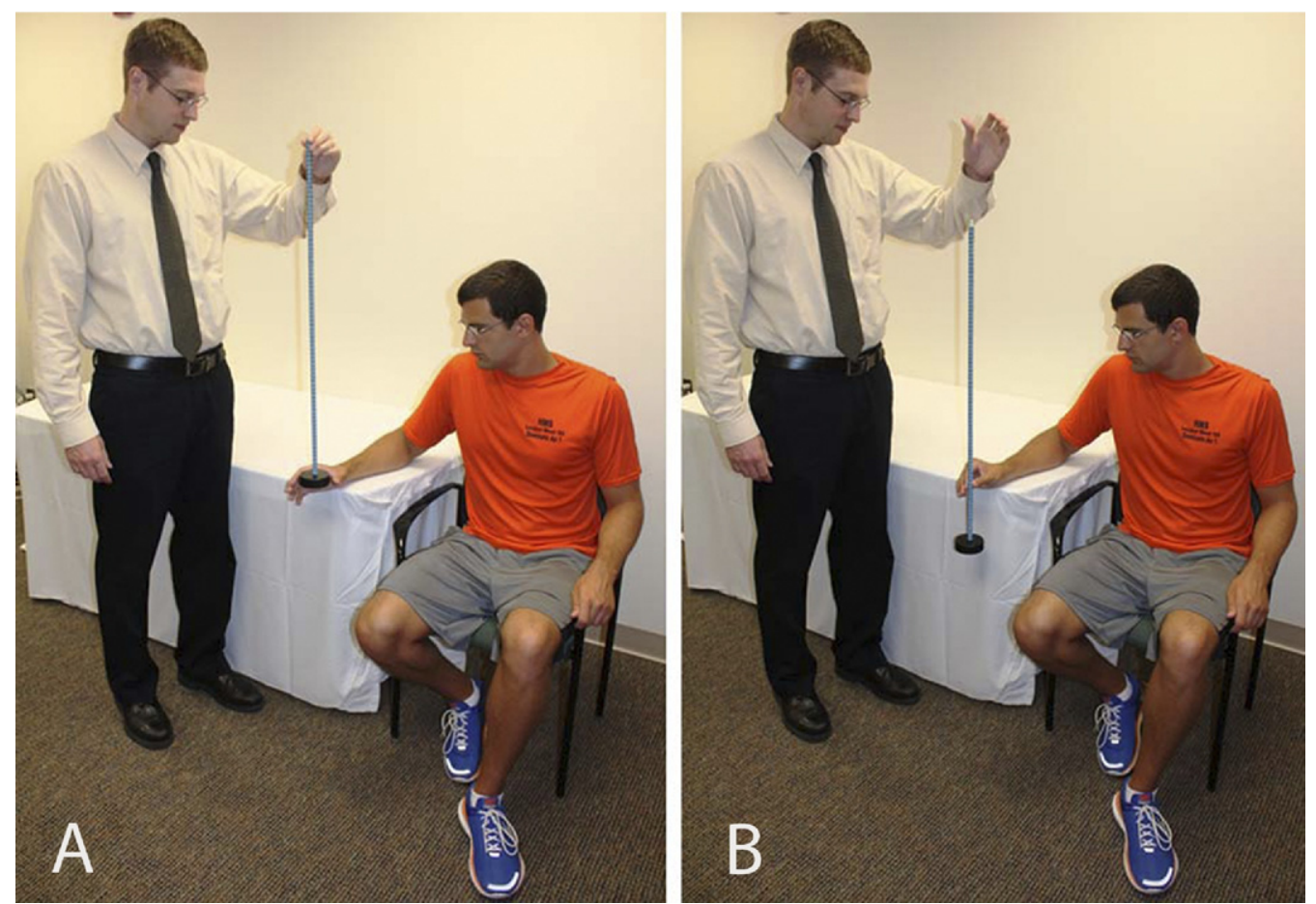

Figure 1. The clinical reaction time procedure. (A) Demonstration of the starting athlete and tester positioning. (B) Demonstration of the post-drop athlete and tester positioning.

peatability $[16,33]$ and sensitivity to the cognitive effects of sports-related concussion [18] are well described in the literature, and the program generally has been accepted by the sports medicine community for use as a concussion assessment tool. The CogState-Sport program performs a series of integrity checks on each test aimed at detecting poor effort or understanding of the test protocol on the part of the test taker in an effort to ensure valid baseline to after-injury comparisons. The simple RT component of the test battery requires the athlete to depress a computer key as rapidly as possible in response to a playing card displayed on the monitor turning face-up. Mean (SD) baseline $\mathrm{RT}_{\text {comp }}$ values (in ms) were calculated for each athlete from their raw CogState-Sport simple RT data.

During the 2-year study period, 9 student athletes sustained a physician-diagnosed concussion and repeated $\mathrm{RT}_{\text {clin }}$ and $\mathrm{RT}_{\text {comp }}$ assessments within 72 hours of injury. The after-injury $\mathrm{RT}_{\text {clin }}$ and $\mathrm{RT}_{\text {comp }}$ tests were performed by using an identical protocol to that used for baseline testing. Mean (SD) after-injury $\mathrm{RT}_{\text {clin }}$ and $\mathrm{RT}_{\text {comp }}$ values (in $\mathrm{ms}$ ) were again calculated, as were the changes in $\mathrm{RT}_{\text {clin }}$ and $\mathrm{RT}_{\text {comp }}$ compared with each athlete's own preseason baseline value. Change scores were calculated as afterinjury value minus baseline value, such that decline is represented by a positive value and improvement is represented by a negative value.

\section{Statistical Analysis}

Given the small sample size, a Wilcoxon signed rank test was used to compare baseline and after-injury RT test performances. Effect sizes for $\mathrm{RT}_{\text {clin }}$ and $\mathrm{RT}_{\text {comp }}$ were described by using Cohen's d. Although the small sample size precluded any formal subgroup analyses, changes in $\mathrm{RT}_{\text {clin }}$ and $\mathrm{RT}_{\text {comp }}$ were descriptively compared or plotted based on history of concussion, presence or absence of loss of consciousness and posttraumatic amnesia, and concussion symptom scores when using the total SCAT postconcussion symptom score as well as the "feeling slowed down" SCAT subscore.

\section{RESULTS}

Demographics, concussion histories, basic injury characteristics, and SCAT scores at the time of after-injury assessment for the 9 athletes with concussion are presented in Table 1 . Compared with their own baseline results, after-injury $\mathrm{RT}_{\text {clin }}$ and $\mathrm{RT}_{\text {comp }}$ values were prolonged in $8(89 \%)$ and $5(56 \%)$ of the 9 athletes with concussion, respectively (Table 2). On average, $\mathrm{RT}_{\text {clin }}$ was $26 \mathrm{~ms}$ slower after concussion $(P=.050)$ and $\mathrm{RT}_{\text {comp }}$ was $215 \mathrm{~ms}$ slower $(P=.214)$. The effect sizes for $\mathrm{RT}_{\text {clin }}$ and $\mathrm{RT}_{\text {comp }}$ were 1.03 and 2.20, respectively.

There appears to be a trend of greater after-injury change in $\mathrm{RT}_{\text {clin }}$ in those athletes who have had more prior concussions compared with those who have not; however, no sim- 
Table 1. Concussion history and injury characteristics for the 9 athletes with concussion

\begin{tabular}{|c|c|c|c|c|c|c|c|}
\hline $\begin{array}{c}\text { Athlete } \\
\text { no. }\end{array}$ & Sport & $\begin{array}{l}\text { Age }(y) \text {, } \\
\text { Gender }\end{array}$ & $\begin{array}{c}\text { Prior } \\
\text { Concussions }\end{array}$ & LOC & PTA & $\begin{array}{l}\text { SCAT: Slowed } \\
\text { Down Score* }\end{array}$ & $\begin{array}{c}\text { SCAT: Total } \\
\text { Score }^{\dagger}\end{array}$ \\
\hline 1 & FB & $22, \mathrm{M}$ & 1 & Yes & No & 1 & 22 \\
\hline 2 & $\mathrm{SOC}$ & $20, F$ & 2 & No & No & 2 & 26 \\
\hline 4 & FB & $19, \mathrm{M}$ & 0 & No & No & 4 & 25 \\
\hline 5 & FB & $20, \mathrm{M}$ & 0 & No & No & 1 & 31 \\
\hline 6 & FB & $20, \mathrm{M}$ & 2 & No & No & 3 & 62 \\
\hline 9 & $\mathrm{FB}$ & $21, \mathrm{M}$ & 1 & No & Yes & 2 & 26 \\
\hline
\end{tabular}

LOC $=$ loss of consciousness; PTA $=$ posttraumatic amnesia; SCAT $=$ Sideline Concussion Assessment Tool; FB $=$ American football, SOC $=$ soccer.

*Each postconcussion SCAT symptom is graded on a 0-6 scale, with 0 representing absence of a symptom and scores of 1-6 representing mild-severe presence of a symptom.

${ }^{\dagger}$ The SCAT postconcussion symptom scale is composed of 24 symptoms and, therefore, has a possible range of 0-144.

ilar trend is apparent for $\mathrm{RT}_{\text {comp }}$ (Table 3). The changes in $\mathrm{RT}_{\text {clin }}$ and $\mathrm{RT}_{\text {comp }}$ in the athlete whose concussion was associated with loss of consciousness do not appear to differ from those of the other 8 athletes (Table 3). There does appear to be a trend toward greater after-injury change in $\mathrm{RT}_{\text {clin }}$ and $\mathrm{RT}_{\text {comp }}$ in the 3 athletes whose concussions were associated with posttraumatic amnesia compared with the 6 athletes whose injuries were not (Table 3). Changes in $\mathrm{RT}_{\text {clin }}$ and $\mathrm{RT}_{\text {comp }}$ are plotted against (1) total SCAT postconcussion symptom scores and (2) the "feeling slowed down" SCAT subscore, the only apparent trend is between $\mathrm{RT}_{\text {clin }}$ and the "feeling slowed down" SCAT subscore (Figure 2).

\section{DISCUSSION}

This study's primary aim was to determine the effect of concussion on $\mathrm{RT}_{\text {clin. }}$. In this sample of 9 athletes with concussion, $\mathrm{RT}_{\text {clin }}$ was, on average, $13.5 \%$ slower after injury compared with the athlete's own preseason baseline. The associated effect size of 1.03, which represents the magnitude of the difference between the athletes' baseline and afterinjury $\mathrm{RT}_{\text {clin }}$ performances proportional to the variability in $\mathrm{RT}_{\text {clin }}$, is generally considered to be large [34]. Only one of the athletes with concussion demonstrated a faster $\mathrm{RT}_{\text {clin }}$ after injury. Given that this athlete was also faster on $\mathrm{RT}_{\text {comp }}$ after injury, it is possible that this particular concussion did not cause a slowed RT. Although concussions do typically result in slower RTs, every concussion is unique, and this phenomenon is not universal. Consistent with this, the athlete rated the subjective symptom of "feeling slowed down" as a 1 on his after-injury SCAT, which suggests that he perceived minimal psychomotor slowing. An alternative explanation is that this athlete did not perform optimally during his baseline test sessions, a possibility supported by the observation that his baseline $\mathrm{RT}_{\text {clin }}$ and $\mathrm{RT}_{\text {comp }}$ values were both among the slowest in our sample. Overall, these findings suggest that $\mathrm{RT}_{\text {clin }}$, in fact, is sensitive to the expected prolongation of RT after concussion.

A secondary study aim was to contrast the effect of concussion on $\mathrm{RT}_{\text {clin }}$ and $\mathrm{RT}_{\text {comp. Although } \mathrm{RT}_{\text {clin }} \text { was more }}$ frequently prolonged than $\mathrm{RT}_{\text {comp }}$, the average magnitude of change was greater for $\mathrm{RT}_{\text {comp }}$, as was its variability. This effect was driven by 2 athletes whose after-injury $\mathrm{RT}_{\text {comp }}$ values were highly variable and very slow. Both of these athletes failed at least one of the CogState-Sport integrity

Table 2. $R T_{\text {clin }}$ and $R T_{\text {comp }}$ summary data for the 9 athletes with concussion

\begin{tabular}{|c|c|c|c|c|c|c|}
\hline $\begin{array}{c}\text { Athlete } \\
\text { no. }\end{array}$ & $\begin{array}{l}\text { Baseline } \mathbf{R T}_{\text {clin }} \\
\text { mean (SD), ms }\end{array}$ & $\begin{array}{c}\text { After-injury } \mathbf{R T}_{\text {clin }} \\
\text { mean (SD), ms }\end{array}$ & $\Delta \mathbf{R T}_{\text {clin }}$ & $\begin{array}{l}\text { Baseline RT } \\
\text { mean (SD), } \mathrm{ms}\end{array}$ & $\begin{array}{l}\text { After-injury RT } \\
\text { mean (SD) } \mathrm{ms}\end{array}$ & $\Delta \mathbf{R T}_{\text {comp }}$ \\
\hline 1 & $180(15)$ & $204(20)$ & 24 & $275(80)$ & $260(39)$ & -15 \\
\hline 2 & 195 (20) & $228(25)$ & 33 & $256(50)$ & $1128(360)$ & 872 \\
\hline 3 & $182(22)$ & $200(31)$ & 18 & $224(28)$ & $248(36)$ & 24 \\
\hline 4 & $208(21)$ & $238(23)$ & 30 & $198(36)$ & 317 (135) & 119 \\
\hline 5 & $217(24)$ & $186(46)$ & -31 & $274(212)$ & $248(37)$ & -26 \\
\hline 6 & $200(32)$ & $218(38)$ & 18 & 301 (137) & 285 (69) & -16 \\
\hline 7 & $204(14)$ & $251(17)$ & 47 & $209(23)^{\star}$ & $229(30)$ & 20 \\
\hline 8 & $156(15)$ & 249 (34) & 93 & $220(54)$ & 1189 (324) & 969 \\
\hline 9 & $191(29)$ & $201(43)$ & 10 & $263(57)$ & $257(51)$ & -6 \\
\hline Average & $193(21)$ & $219(31)$ & 26 & $247(75)$ & $462(120)$ & 215 \\
\hline
\end{tabular}

$\mathrm{RT}_{\text {clin }}=$ clinically measured reaction time; $\mathrm{RT}_{\text {comp }}=$ computerized reaction time measure.

*Athlete 7 did not have a valid baseline test session from the season of injury, so the prior year's baseline was used. 
Table 3. After-injury changes in $R T_{\text {clin }}$ and $R T_{\text {comp }}$ in subgroups of athletes based on prior concussion history, presence of LOC, and presence of PTA

\begin{tabular}{llcc}
\hline $\begin{array}{c}\text { Athlete } \\
\text { Subgroup }\end{array}$ & $\mathbf{n}$ & $\boldsymbol{\Delta} \mathbf{R T}_{\text {clin }}, \mathbf{m s}$ & $\boldsymbol{\Delta} \mathbf{R T}_{\text {comp }}, \mathbf{m s}$ \\
\hline Prior concussions & & & \\
0 & 4 & 16 & 34 \\
1 & 2 & 17 & -11 \\
2 & 2 & 26 & 428 \\
3 & 1 & 93 & 969 \\
LOC & & & \\
Yes & 1 & 24 & -15 \\
No & 8 & 27 & 245 \\
PTA & & & \\
Yes & 3 & 40 & 329 \\
No & 6 & 20 & 159 \\
\hline
\end{tabular}

$\mathrm{RT}_{\text {clin }}=$ clinically measured reaction time; $\mathrm{RT}_{\text {comp }}=$ computerized reaction time measure; $\mathrm{LOC}=$ loss of consciousness; PTA = posttraumatic amnesia.

checks during these after-injury evaluations, and close inspection of the full reports for these test sessions revealed that their performance was very slow throughout all 4 of the CogState-Sport test modules. These findings are unlikely to be entirely attributable to cognitive slowing as a result of the athletes' concussions. One possible explanation is distraction due to headache or some other postconcussion symptom. Another possible explanation is poor motivation because the athletes already recognized that they had sustained a concussion and would not be immediately cleared to return to play regardless of their performance on that particular after-injury test. We do not believe that these athletes had any problem understanding the computerized test procedure because both had completed valid baseline tests at the beginning of the season during which they sustained their concussions. It is interesting to note that, although both of these athletes did have slower postconcussion $\mathrm{RT}_{\text {clin }}$ performances, the magnitude of slowing is much smaller for $\mathrm{RT}_{\text {clin }}$ than for $\mathrm{RT}_{\text {comp }}$. Potential reasons for this difference include the fact that $\mathrm{RT}_{\text {clin }}$ is bound by a maximum value of approximately 400 $\mathrm{ms}$, the amount of time that elapses between release of the device and contact with the ground, whereas $\mathrm{RT}_{\text {comp }}$ has no upper limit. Also, athletes may more consistently attend to $\mathrm{RT}_{\text {clin }}$ testing because it involves direct interaction between the athlete and the examiner, is an intrinsically more-interesting task, and lasts only 3-5 minutes. In contrast, $\mathrm{RT}_{\text {comp }}$ testing does not involve human interaction, requires a more mundane task (pressing a computer keyboard key), and is part of a longer 15-minute test battery.

Although no definitive inferences can be drawn from subgroup analysis of this small sample of athletes with concussion, 3 interesting trends are noted. First, the magnitude of after-injury change in $\mathrm{RT}_{\text {clin }}$ appears to increase as an athlete's number of prior concussions increases. Prior studies, including our own previous work that used $\mathrm{RT}_{\text {clin }}$, have failed to identify a relationship between an athlete's prior concussion history and baseline RT measures $[28,35]$ or after-injury change in RT [36]; however, a history of 2 or more concussions has been associated with slower recovery of RT impairment after sports-related concussion [37]. Second, the after-injury changes in both $\mathrm{RT}_{\text {clin }}$ and $\mathrm{RT}_{\text {comp }}$ appear to be greater in the subset of athletes who experienced posttraumatic amnesia compared with those who did not. Although we are unaware of any studies that directly compare the presence or duration of posttraumatic amnesia with after-injury changes in RT, this finding is noteworthy because previous studies have identified an association between posttraumatic amnesia and various concussion severity outcome measures [25,38-41], Third, as illustrated in Figure 2, there appears to be a positive relationship between the subjective rating by an athlete with concussion of "feeling slowed down" and the after-injury change in $\mathrm{RT}_{\text {clin }}$. If these apparent trends can be replicated in a larger sample of athletes with sportsrelated concussion, then these findings would further support the face and construct validity of $\mathrm{RT}_{\text {clin }}$.

The results of this preliminary $\mathrm{RT}_{\text {clin }}$ study are consistent with existing literature that reports the detrimental shortterm effects of sports-related concussion on computer-based RT measures. For example, Makdissi et al [20] reported CogSport-based data obtained within 3 days of concussion in a series of 6 male Australian Rules football players that showed an average increase in simple RT from $292.4 \pm 88.6$ ms at baseline to $339.9 \pm 128.7 \mathrm{~ms}$ after injury. Similarly, Warden et al [21] reported Automated Neuropsychological Assessment Metrics-based data at baseline and 4 days after a boxing concussion in 14 male military cadets that demonstrated an average increase in simple RT, from $254 \pm 33 \mathrm{~ms}$ to $342 \pm 36 \mathrm{~ms}$. Larger studies have reported similar trends in short-term computerized RT performance by using composite or throughput scores. Examples are McClincy et al [24], who reported impaired Immediate Post-Concussion

\section{Change in $\mathbf{R T}_{\text {clin }}$ vs. "slowed down" SCAT score}

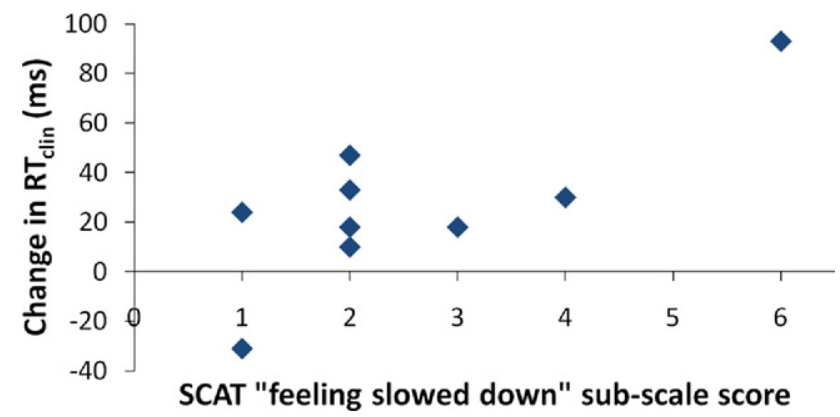

Figure 2. Plot that illustrates the relationship between afterinjury change in clinically measured reaction time ( $\left.R T_{\text {clin }}\right)$ and the athlete's self-reported rating of "feeling slowed down" on the Sideline Concussion Assessment Tool (SCAT) at the time of after-injury testing. 
Assessment and Cognitive Test RT composite scores in 104 high school and collegiate athletes at 2 days and 1 week after concussion, with return to baseline 2 weeks after injury, and McCrea et al [22], who reported impaired Automated Neuropsychological Assessment Metrics RT throughput scores in 28 high school and collegiate football players immediately after concussion, with a return to baseline when tested at postinjury days 8 and 45 . Although this format of data presentation does not permit direct comparison with our $\mathrm{RT}_{\text {clin }}$ results, these findings are consistent with the trend of impaired $\mathrm{RT}_{\text {clin }}$ performance in our sample of athletes in the days immediately after concussion.

This was a small, unblinded study, and these are potential sources of bias. Therefore, a larger, blinded study is necessary before firm conclusions are drawn. Follow-up studies should more precisely standardize the timing of after-injury testing and should test at multiple postinjury time points to monitor trends in $\mathrm{RT}_{\text {clin }}$ performance during recovery. In addition, athletes without concussion need to be tested concurrently to control for the effects of postcompetition fatigue and nonconcussion-inducing head contact. Despite the absence of control data, it is reasonable to hypothesize that repeated $\mathrm{RT}_{\text {clin }}$ testing in uninjured athletes would result in faster RTs given other work that showed that baseline $\mathrm{RT}_{\text {clin }}$ significantly decreased (became faster) by $11 \mathrm{~ms}$ in 102 uninjured college athletes over 2 seasons [30].

\section{CONCLUSION}

We do not propose that $\mathrm{RT}_{\text {clin }}$ should replace computerized testing in athletes who have access to it but rather that $\mathrm{RT}_{\text {clin }}$ may complement computerized tests. However, should further work continue to support the sensitivity of $\mathrm{RT}_{\text {clin }}$ to concussion, then its simplicity, portability, and low cost all represent advantages over computerized test batteries. These advantages may allow $\mathrm{RT}_{\text {clin }}$ to serve as a true sideline tool during the initial evaluation of an athlete with suspected concussion, a setting in which computerized testing is impractical. These attributes should also make $\mathrm{RT}_{\text {clin }}$ available to a broader population of athletes, particularly youth athletes who sustain the majority of concussions [42] but usually have limited financial resources. Used with other clinical concussion-assessment tools, $\mathrm{RT}_{\text {clin }}$ has the potential to become a useful part of the sports medicine practitioner's multifaceted concussion assessment battery.

\section{ACKNOWLEDGMENTS}

We are grateful for the assistance of Mr Steve Nordwall, head athletic trainer, Eastern Michigan University, and his athletic training staff, as well as Dr David Darby, chief medical officer, CogState Ltd.

\section{REFERENCES}

1. Langlois JA, Rutland-Brown W, Wald MM. The epidemiology and impact of traumatic brain injury: a brief overview. J Head Trauma Rehabil 2006;21:375-378.

2. Powell JW, Barber-Foss KD. Traumatic brain injury in high school athletes. JAMA 1999;282:958-963.

3. Gessel LM, Fields SK, Collins CL, Dick RW, Comstock RD. Concussions among United States high school and collegiate athletes. J Athl Train 2007:42:495-503.

4. Collins MW, Lovell MR, McKeag DB. Current issues in managing sports-related concussion. JAMA 1999;282:2283-2285.

5. Kelly JP. Traumatic brain injury and concussion in sports. JAMA 1999;282:989-991

6. McKee AC, Cantu RC, Nowinski CJ, et al. Chronic traumatic encephalopathy in athletes: progressive tauopathy after repetitive head injury. J Neuropathol Exp Neurol 2009;68:709-735.

7. Gavett BE, Stern RA, Cantu RC, Nowinski CJ, McKee AC. Mild traumatic brain injury: a risk factor for neurodegeneration. Alzheimers Res Ther 2010;2:18.

8. De Beaumont $\mathrm{L}$, Theoret $\mathrm{H}$, Mongeon $\mathrm{D}$, et al. Brain function decline in healthy retired athletes who sustained their last sports concussion in early adulthood. Brain 2009;132:695-708.

9. Guskiewicz KM, Bruce SL, Cantu RC, et al. National Athletic Trainers' Association position statement: management of sport-related concussion. J Athl Train 2004;39:280-297.

10. McCrory P, Meeuwisse W, Johnston K, et al. Consensus statement on Concussion in Sport 3rd International Conference on Concussion in Sport held in Zurich, November 2008. Clin J Sport Med 2009;19:185200.

11. Halstead ME, Walter KD. American Academy of Pediatrics. Clinical report: sport-related concussion in children and adolescents. Pediatrics 2010;126:597-615.

12. Iverson GL, Lovell MR, Collins MW. Validity of ImPACT for measuring processing speed following sports-related concussion. J Clin Exp Neuropsychol 2005;27:683-689.

13. Collie A, Maruff P, Darby D, Makdissi M, McCrory P, McStephen M. CogSport. In: Echemendia RJ, ed. Sports Neuropsychology: Assessment and Management of Traumatic Brain Injury. New York, NY: Guildford Publications; 2006:240-262.

14. Cernich A, Reeves D, Sun W, Bleiberg J. Automated Neuropsychological Assessment Metrics sports medicine battery. Arch Clin Neuropsychol 2007;22:S101-S114.

15. Erlanger D, Saliba E, Barth J, Almquist J, Webright W, Freeman J. Monitoring resolution of postconcussion symptoms in athletes: preliminary results of a web-based neuropsychological test protocol. J Athl Train 2001;36:280-287.

16. Collie A, Maruff P, Makdissi M, McCrory P, McStephen M, Darby D. CogSport: reliability and correlation with conventional cognitive tests used in postconcussion medical evaluations. Clin J Sport Med 2003; 13:28-32.

17. Lau B, Lovell MR, Collins MW, Pardini J. Neurocognitive and symptom predictors of recovery in high school athletes. Clin J Sport Med 2009; 19:216-221.

18. Collie A, Makdissi M, Maruff P, Bennell K, McCrory P. Cognition in the days following concussion: comparison of symptomatic versus asymptomatic athletes. J Neurol Neurosurg Psychiatry 2006;77:241-245.

19. Lovell MR, Collins MS, Fu FH, et al. Neuropsychological testing in sports: past, present, and future. Br J Sports Med 2001;35:373.

20. Makdissi M, Collie A, Maruff $P$, et al. Computerised cognitive assessment of concussed Australian Rules footballers. Br J Sports Med 2001 35:354-360.

21. Warden DL, Bleiberg J, Cameron KL, et al. Persistent prolongation of simple reaction time in sports concussion. Neurology 2001;57:524526. 
22. McCrea M, Prichep L, Powell MR, Chabot R, Barr WB. Acute effects and recovery after sport-related concussion: a neurocognitive and quantitative brain electrical activity study. J Head Trauma Rehabil 2010;25: 283-292.

23. Makdissi M, Darby D, Maruff P, Ugoni A, Brukner P, McCrory PR. Natural history of concussion in sport: markers of severity and implications for management. Am J Sports Med 2010;38:464-471.

24. McClincy MP, Lovell MR, Pardini J, Collins MW, Spore MK. Recovery from sports concussion in high school and collegiate athletes. Brain Inj 2006;20:33-39.

25. Collins MW, Field M, Lovell MR, et al. Relationship between postconcussion headache and neuropsychological test performance in high school athletes. Am J Sports Med 2003;31:168-173.

26. Broglio SP, Sosnoff JJ, Ferrara MS. The relationship of athlete-reported concussion symptoms and objective measures of neurocognitive function and postural control. Clin J Sport Med 2009;19:377-382.

27. Broglio SP, Macciocchi SN, Ferrara MS. Neurocognitive performance of concussed athletes when symptom free. J Athl Train 2007;42:504-508.

28. Eckner JT, Kutcher JS, Richardson JK. Pilot evaluation of a novel clinical test of reaction time in National Collegiate Athletic Association Division I football players. J Athl Train 2010;45:327-332.

29. Eckner JT, Whitacre RD, Kirsch NK, Richardson JK. Evaluating a clinical measure of reaction time: an observational study. Percept Mot Skills 2009;108:717-720.

30. Eckner JT, Kutcher JS, Richardson JK. Between season test-retest reliability of clinically measured reaction time in NCAA Division I collegiate athletes. J Athl Train 2011. In press.

31. Eckner JT, Lipps DB, Kim H, Richardson JK, Ashton-Miller JA. Can a clinical test of reaction time predict a functional head-protective response? Med Sci Sports Exerc 2010 Aug 2 [Epub ahead of print].

32. McCrory $P$, Johnston $K$, Meeuwisse $W$, et al. Summary and agreement statement of the 2nd International Conference on Concussion in Sport, Prague 2004. Br J Sports Med 2005;39:196-204.

33. Collie A, Maruff P, Darby DG, McStephen M. The effects of practice on the cognitive test performance of neurologically normal individuals assessed at brief test-retest intervals. J Int Neuropsychol Soc 2003; 9:419-428.

34. Cohen J. Statistical Power Analysis for the Behavioral Sciences. 2nd ed. Hillsdale, NJ: Lawrence Erlbaum Associates; 1988.

35. Covassin $T$, Elbin R, Kontos A, Larson E. Investigating baseline neurocognitive performance between male and female athletes with a history of multiple concussion. J Neurol Neurosurg Psychiatry 2010;81:597601.

36. Iverson GL, Gaetz M, Lovell MR, Collins MW. Cumulative effects of concussion in amateur athletes. Brain Inj 2004;18:433-443.

37. Covassin T, Stearne D, Elbin R. Concussion history and postconcussion neurocognitive performance and symptoms in collegiate athletes. J Athl Train 2008;43:119-124.

38. McCrea M, Kelly JP, Randolph C, Cisler R, Berger L. Immediate neurocognitive effects of concussion. Neurosurgery 2002;50:10321040 .

39. Pellman EJ, Viano DC, Casson IR, Arfken C, Powell J. Concussion in professional football: injuries involving 7 or more days out—part 5 . Neurosurgery 2004;55:1100-1119.

40. Collins MW, Iverson GL, Lovell MR, McKeag DB, Norwig J, Maroon J. On-field predictors of neuropsychological and symptom deficit following sports-related concussion. Clin J Sport Med 2003;13:222-229.

41. Lovell MR, Collins MW, Iverson GL, et al. Recovery from mild concussion in high school athletes. J Neurosurg 2003;98:296-301.

42. CDC. Nonfatal traumatic brain injuries from sports and recreation activities: United States, 2001-2005. MMWR Morb Mortal Wkly Rep 2007;56:733-737.

This CME activity is designated for 1.0 AMA PRA Category 1 Credit ${ }^{\mathrm{TM}}$ and can be completed online at me.aapmr.org. Log on to www.me.aapmr.org, go to Lifelong Learning (CME) and select Journal-based CME from the drop down menu. This activity is FREE to AAPMER members and $\$ 25$ for non-members.

\section{CME Question}

In the pilot study by Eckner et al, clinical reaction time $\left(\mathrm{RT}_{\text {clin }}\right)$ demonstrated which of the following characteristics when compared to computerized reaction time ( $\left(\mathrm{T}_{\text {comp }}\right)$ ?

a. $\mathrm{RT}_{\text {clin }}$ was more variable.

b. RT clin was prolonged in more athletes.

c. RT $T_{\text {clin }}$ was shortened post-injury in all athletes.

d. RT $_{\text {clin }}$ did not measure pre-and post-concussion change. 